\title{
Molecular and functional analysis of pTAV320, a repABC-type replicon of the Paracoccus versutus composite plasmid pTAV1
}

\author{
Dariusz Bartosik, Jadwiga Baj and Miroslawa Wlodarczyk
}

Author for correspondence: Dariusz Bartosik. Tel/Fax: +48 2282689 82. e-mail : bartosik@plearn.edu.pl

Department of Bacterial Genetics, Institute of Microbiology, Warsaw University, Nowy Swiat 67, 00-046 Warsaw, Poland

\begin{abstract}
The second replicator region of the native plasmid pTAV1 of Paracoccus versutus has been identified thus proving the composite nature of this replicon. The minimal replicon designated pTAV320 (4.3 kb) was cloned and sequenced. pTAV320 encodes three putative proteins - RepA, RepB and RepC. This replicator region shows strong structural and functional similarity to repABC-type replicons found in several Agrobacterium and Rhizobium plasmids. The origin of replication appears to be localized within the coding sequence of the repC gene. RepC was shown to be essential for replication. RepA and RepB were necessary for stable maintenance of the plasmid, which implies a role in active partitioning. The presence of the complete sequence of PTAV320 (in its non-replicative form) could stabilize in cis pTAV202, a minireplicon derived from the other pTAV1 replicator region. Deletions introduced into the repC gene abolished the 'stabilizing' activity of pTAV320, suggesting that the centromere-like sequence, necessary for partitioning, might be localized within this gene. The two replicator regions of pTAV1 (pTAV320 and PTAV202) expressed incompatibility towards the parental plasmid but were compatible in trans in P. versutus cells. The pTAV320 replicon can be maintained in several Paracoccus, Agrobacterium, Rhizobium and Rhodobacter strains in addition to $P$. versutus.
\end{abstract}

Keywords: Paracoccus versutus, pTAV1 composite plasmid, repABC replicon, plasmid replication and stability, minimal replicon

\section{INTRODUCTION}

Paracoccus versutus, previously Thiobacillus versutus, is a Gram-negative, facultatively chemolithoautotrophic soil bacterium belonging to the $\alpha$ subclass of the Proteobacteria. $P$. versutus UW1 harbours a large (107 kb), low-copy-number, cryptic plasmid, pTAV1 (Bednarska et al., 1983), which is stably maintained in its bacterial host. The replication system of this plasmid has been identified by construction of a mini-replicon, pTAV202 (Bartosik et al., 1995), and its shortened form pTAV203 (Bartosik et al., 1997a), which confers all functions sufficient for autonomous replication in $P$. versutus. pTAV203 carries a $3 \cdot 1 \mathrm{~kb}$ SphI-PstI restriction fragment of pTAV1 linked to the $\mathrm{Km}^{\mathrm{r}}$ cassette derived from pUC4-K. The nucleotide sequence of this replicator region (GenBank accession no. U42228) revealed the

The GenBank accession number for the sequence reported in this paper is U60522. presence of two genes of the same transcriptional polarity encoding two putative proteins: RepC' $(45$ $\mathrm{kDa})$ and $\operatorname{RepX}(26 \mathrm{kDa})$. Further analysis of pTAV203 showed that under certain conditions the rep $C^{\prime}$ gene itself was able to provide replication function (Bartosik et al., 1997b). Therefore, this proved that RepC' is the major replication initiator protein and that the origin of replication is located along its coding sequence.

The replication system of pTAV203 appears to be related to several plasmids commonly found in Gramnegative soil bacteria. $\mathrm{RepC}^{\prime}$ shares statistically significant homology with the RepC replication protein of pRmeGR4a of Sinorhizobium meliloti (Mercado-Blanco $\&$ Olivares, 1994) and RepC of repABC-type replicons, e.g. pRiA4b of Agrobacterium rhizogenes (Nishiguchi et al., 1987) and pRL8JI of Rhizobium leguminosarum (Turner \& Young, 1995). Despite the homology of RepC proteins, no other structural similarities between pTAV203 and other known replicons were found. 
The mini-replicon pTAV203 is not stably maintained in $P$. versutus cells. The stable maintenance of pTAV202 or pTAV203 and probably entire pTAV1 depended on a specific $14 \mathrm{~kb}$ EcoRI-Bcll restriction fragment of the parental plasmid. This fragment when introduced in cis could fully stabilize pTAV203, but also carried a second replication system of pTAV1. Although it is suggested that many large plasmids are composite replicons (Helinski et al., 1996), few examples have been described. These are mainly Escherichia coli plasmids belonging to the IncF incompatibility groups (Bergquist et al., 1982) and the Streptococcus plasmid pAM $\alpha 1$ (Perkins \& Youngman, 1983).

We report here results of an experimental approach designed to characterize the second replicator region of pTAV1, included in the mini-replicon pTAV320. Molecular analysis of both replication systems will enable the study of possible interactions between them in the parental composite plasmid pTAV1.

\section{METHODS}

Bacterial strains, plasmids and growth conditions. Bacterial strains and plasmids used in this study are listed in Table 1. Cultures were grown in Luria broth (LB) (Sambrook et al., 1989) at $30^{\circ} \mathrm{C}$ (P. versutus, Agrobacterium tumefaciens and Rhodobacter sphaeroides) or $37^{\circ} \mathrm{C}$ (E. coli) and in TY medium (Beringer, 1974) at $30^{\circ} \mathrm{C}$ (Rhizobium etli and Rhiz. leguminosarum $\rangle$. Antibiotics included in the media were used at the following final concentrations $\left(\mu \mathrm{g} \mathrm{ml}^{-1}\right)$ : kanamycin, 50; streptomycin, 100 for Rhiz. etli and 400 for Rhiz. leguminosarum; rifampicin, 50; tetracycline, 1 for $R$ hod. sphaeroides, 3 for P. versutus, 5 for Rhiz. leguminosarum, 10 for Rhiz. etli and 20 for E. coli.

DNA manipulations. Plasmid DNA was isolated according to Birnboim \& Doly (1979) and purified by $\mathrm{CsCl} /$ ethidium bromide gradient centrifugation. Cloning experiments, digestion with restriction enzymes, ligation, treatment with the Klenow fragment of DNA polymerase I and agarose gel electrophoresis were conducted in accordance with standard procedures as described by Sambrook et al. (1989). All enzymes were purchased from either Promega or Boehringer Mannheim. DNA restriction fragments were purified from agarose with the DNA-Gel-Out kit (DNA Gdansk). For Southern hybridization (Sambrook et al., 1989), DNA probes were labelled with digoxigenin (Boehringer Mannheim). Hybridization and visualization of hybridization products were carried out as recommended by the supplier.

PCR amplification. PCR was used to amplify the repC gene. Oligonucleotides were synthesized by Universal DNA. The $5^{\prime}$ oligonucleotide contained the nucleotide sequence of the amino-terminus of $\mathrm{RepC}$ (including a putative ribosomebinding site), including a Pst I restriction endonuclease site (underlined): 5' AACTGCAGCGTGAATCACATCATGGAGTA 3'. The 3' oligonucleotide contained the nucleotide sequence encoding the carboxyl-terminus of RepC, including a PstI restriction site (underlined): $5^{\prime}$ AACTGCAGGAGACTTAACAGCTGTTATG $3^{\prime}$. The amplification was performed in Thermocycler Hot-Shot 18 (DNA Gdansk) using the above synthetic oligonucleotides, Thermus aquaticus polymerase (DNA Gdansk) and pTAV1 as a template DNA. The amplification cycle used was $94^{\circ} \mathrm{C}$ for $5 \mathrm{~min}$, followed by 30 cycles of $94^{\circ} \mathrm{C}$ for $30 \mathrm{~s}, 56^{\circ} \mathrm{C}$ for $30 \mathrm{~s}, 72^{\circ} \mathrm{C}$ for $2 \mathrm{~min}$; the last cycle was followed by an additional extension step of $5 \mathrm{~min}$.

DNA sequencing and analysis. The nucleotide sequence was determined in the DNA Sequencing and Oligonucleotides Synthesis Laboratory, Institute of Biochemistry and Biophysics, Polish Academy of Science, using a terminator sequencing kit and automatic sequencer (Alf Pharmacia). The two PstI fragments of pTAV320 $(2 \cdot 1$ and $2 \cdot 2 \mathrm{~kb})$ were cloned into $E$. coli pBGS18 vector and the inserts were sequenced starting with universal and reverse primers and then with primers complementary to the previously determined sequence. Sequence analysis and comparison searches through the databases were performed with TFASTA, BLAST and GAP (Wisconsin Genetics Computer Group Sequence Analysis Software Package; GCG version 8.1). The alignment of the sequences was performed with the PILEUP program (GCG software). Phylogenetic analysis of RepC sequences was performed with the neighbour-joining method included in the PHYLIP package (Felsenstein, 1993).

Electroporation and transformation. Electroporation was carried out at $2500 \mathrm{~V}, 25 \mu \mathrm{F}$ and $200 \Omega$ (for E. coli) or $400 \Omega$ (for P. versutus, Paracoccus denitrificans and A. tumefaciens) in a gene pulser apparatus (Bio-Rad) according to a modified Bio-Rad procedure (Wlodarczyk et al., 1994). Electrotransformants were selected on solidified LB medium supplemented with appropriate antibiotics. Competent cells of $E$. coli TG1 were prepared and transformed as described by Kushner (1978).

Triparental mating. The overnight cultures (spun down and washed twice to remove antibiotics) of the donor strain E. coli TG1 carrying the mobilizable recombinant vector based on pABW2 (Bartosik et al., 1997b), suitable recipient strain and E. coli DH5 $\alpha$ carrying the helper plasmid pRK2013 were mixed at a ratio $1: 2: 1$. One hundred microlitres of such a mixture was spread on a plate of solidified TY or LB medium, depending on the recipient strain. After overnight incubation at $30^{\circ} \mathrm{C}$ the bacteria were washed off the plate and suitable dilutions were plated on selective media containing rifampicin or streptomycin, selective markers of the recipient strains, and other appropriate antibiotic (tetracycline or kanamycin) to select transconjugants. Spontaneous resistance of the recipient strains to kanamycin and tetracycline was undetectable under these experimental conditions.

Plasmid stability. Cultures of P. versutus, Rhiz. etli, Rhiz. leguminosarum and Rhod. sphaeroides containing plasmids to be tested were grown in LB medium supplemented with appropriate antibiotics. The late exponential growth phase cells were diluted $10^{-3}$ in LB medium without antibiotics and grown at $30^{\circ} \mathrm{C}$. Cultures were diluted $10^{-3}$ at $24 \mathrm{~h}$ intervals. Samples taken at each dilution step were plated on solidified LB medium for estimation of the number of generations. From these plates 200 colonies were tested with the use of a $\mathrm{Km}^{\mathrm{r}}$ or $T c^{r}$ marker by replica plating. The retention of plasmids, after approximately 30 generations, was determined as the percentage of $\mathrm{Km}^{\mathrm{r}}$ or $\mathrm{Tc}^{\mathrm{r}}$ colonies.

Incompatibility testing. The incompatibility characteristics of two plasmids were examined by electroporation of the plasmid to be tested into recipient bacteria carrying the other plasmid. Transformants were selected for the incoming plasmid or, in the case of analysis of coexistence in trans of the two pTAV1 mini-replicons, for incoming and residing plasmids. The plasmid pattern of the transformants was verified by screening 10 colonies by a rapid alkaline extraction procedure and agarose gel electrophoresis. 
Table 1. Bacterial strains and plasmids used in this work

\begin{tabular}{|c|c|c|}
\hline Strain or plasmid & Relevant characteristics & Reference or source \\
\hline \multicolumn{3}{|l|}{ Strains } \\
\hline \multicolumn{3}{|l|}{ Paracoccus versutus } \\
\hline UW1 & Wild-type (first described as Thiobacillus sp. strain A2) & Taylor \& Hoare (1969) \\
\hline UW225 & Rifr, pTAV1-less derivative of UW1 & Bartosik et al. (1993) \\
\hline Paracoccus denitrificans KL1 & Wild-type, carries native pKL1 (100 kb) & Jordan et al. (1997) \\
\hline Agrobacterium tumefaciens LBA1010 & Carries native pTiB6S3 $(195 \mathrm{~kb})$ & Koekman et al. (1982) \\
\hline Rhizobium etli CE3 & Str ${ }^{\mathrm{r}}$ & Noel et al. (1984) \\
\hline Rhizobium leguminosarum 1062 & Str ${ }^{r}$ & Hirsch et al. (1980) \\
\hline Rhodobacter sphaeroides $2.4 .1 \mathrm{R}$ & Rif derivative of a wild-type 2.4 .1 strain & Suwanto \& Kaplan (1992) \\
\hline \multicolumn{3}{|l|}{ Escherichia coli } \\
\hline TG1 & Host strain for recombinant plasmids & Sambrook et al. (1989) \\
\hline $\mathrm{DH} 5 \alpha$ & Host strain for pRK2013 & Hanahan (1983) \\
\hline \multicolumn{3}{|l|}{ Plasmids } \\
\hline pTAV1 & Wild-type, cryptic, $107 \mathrm{~kb}$ & Bednarska et al. (1983) \\
\hline pTAV110 & $\begin{array}{l}\text { Mini-replicon carrying } 30 \mathrm{~kb} \text { EcoRI fragment of pTAV1 } \\
\text { plus } \mathrm{Km}^{\mathrm{r}} \text { cassette }(1.3 \mathrm{~kb}) \text { derived from pUC4-K }\end{array}$ & Bartosik et al. (1995) \\
\hline pTAV112 & $\begin{array}{l}\text { pTAV110 derivative with } 596 \text { bp BglII deletion covering } \\
\text { part of } r e p C\end{array}$ & This work \\
\hline pTAV202 & $\begin{array}{l}\text { Mini-replicon carrying } 4 \mathrm{~kb} \text { Sall-PstI restriction fragment } \\
\text { of pTAV1 plus } \mathrm{Km}^{\mathrm{r}} \text { cassette derived from pUC4-K }\end{array}$ & Bartosik et al. (1995) \\
\hline pTAV202T & $\begin{array}{l}\mathrm{Tc}^{\mathrm{r}} \text { derivative of pTAV202, } \mathrm{Tc}^{\mathrm{r}} \text { cassette derived from } \\
\text { pBR322 }\end{array}$ & This work \\
\hline pTAV203 & $\begin{array}{l}\text { Mini-replicon containing a } 3.1 \mathrm{~kb} \text { SphI-PstI fragment of } \\
\text { pTAV202 plus } \mathrm{Km}^{r} \text { cassette derived from pUC4-K }\end{array}$ & Bartosik et al. (1997a) \\
\hline pTAV202/18 & $\mathrm{Km}^{\mathrm{r}}$, pBGS18 carrying $4 \mathrm{~kb}$ PstI fragment of pTAV202 & Bartosik et al. (1995) \\
\hline pTAV202/18/16 & $\begin{array}{l}\mathrm{Km}^{\mathrm{r}}, 16 \mathrm{~kb} \text { BclI fragment of pTAV110 cloned into } \\
\text { Bam HI site of pBGS202/18 }\end{array}$ & This work \\
\hline pTAV301 & $\begin{array}{l}\text { pTAV1 mini-derivative composed of } 16 \cdot 1 \mathrm{~kb} \mathrm{BclI} \\
\text { fragment of pTAV110 plus Km }{ }^{r} \text { cassette derived from } \\
\text { pUC4-K }\end{array}$ & This work \\
\hline pTAV310 & $\begin{array}{l}\text { Mini-replicon containing } 9 \mathrm{~kb} \text { Sall fragment of pTAV301 } \\
\text { plus } \mathrm{Km}^{\mathrm{r}} \text { cassette derived from pUC4-K }\end{array}$ & This work \\
\hline pTAV320 & $\begin{array}{l}\text { Minimal replicon composed of two PstI fragments }(2 \cdot 1 \\
\text { and } 2 \cdot 2 \mathrm{~kb} \text { ) of pTAV1 plus } \mathrm{Km}^{\mathrm{r}} \text { cassette derived from } \\
\text { pUC4-K }\end{array}$ & This work \\
\hline pTAV321 & pTAV320 derivative with 32 bp BamHI deletion in repA & This work \\
\hline pTAV322 & $\begin{array}{l}\text { pTAV320 derivative with inserted } \mathrm{Km}^{\mathrm{r}} \text { cassette (from } \\
\text { pUC4-K) into PstI site of repB gene }\end{array}$ & This work \\
\hline pUC4-K & Source of $\mathrm{Km}^{\mathrm{r}}$ cassette & Vieira \& Messing (1982) \\
\hline pBGS18 & $\mathrm{Km}^{\mathrm{r}}$, cloning vector, ColE1 origin of replication & Spratt et al. (1986) \\
\hline pABW2 & $\begin{array}{l}\mathrm{Tc}^{\mathrm{r}} \text {, cloning vector based on } \mathrm{pBGS18}, \mathrm{Tc}^{\mathrm{r}} \text { derived from } \\
\mathrm{Tn} 1721 \text {, oriT of RK2 }\end{array}$ & Bartosik et al. (1997b) \\
\hline $\mathrm{pABW} 320$ & $\begin{array}{l}\mathrm{Tc}^{\mathrm{r}} \mathrm{Km}^{\mathrm{r}} \text {, pABW } 2 \text { derivative containing } 5 \cdot 6 \mathrm{~kb} \text { non- } \\
\text { replicative form of pTAV } 320 \text { linearized by StuI }\end{array}$ & This work \\
\hline pABW 202 & $\begin{array}{l}\mathrm{Tc}^{\mathrm{r}} \text {, pABW2 derivative containing } 4 \mathrm{~kb} \text { Pst } \mathrm{I} \text { replicator } \\
\text { region of pTAV202 }\end{array}$ & This work \\
\hline pABW2021 & $\begin{array}{l}\mathrm{Tc}^{r} \mathrm{Km}^{\mathrm{r}}, \text { pABW } 202 \text { derivative containing } 4 \cdot 3 \mathrm{~kb} \\
\text { Bsu36I-BglII fragment of pTAV320 }\end{array}$ & This work \\
\hline pABW2022 & $\begin{array}{l}\mathrm{Tc}^{r} \mathrm{Km}^{\mathrm{r}} \text {, pABW202 derivative containing } 5.6 \mathrm{~kb} \text { non- } \\
\text { replicative form of pTAV } 320 \text { linearized by StuI }\end{array}$ & This work \\
\hline pABW2023 & $\begin{array}{l}\mathrm{Tc}^{\mathrm{r}} \mathrm{Km}^{\mathrm{r}} \text {, pABW } 202 \text { derivative containing } 5 \mathrm{~kb} \text { BglII } \\
\text { fragment of pTAV } 320\end{array}$ & This work \\
\hline pABW2024 & $\begin{array}{l}\mathrm{Tc}^{\mathrm{r}} \mathrm{Km}^{\mathrm{r}} \text {, pABW } 202 \text { derivative containing } 5.6 \mathrm{~kb} \text { non- } \\
\text { replicative form of pTAV320 linearized by Bsu36I }\end{array}$ & This work \\
\hline $\mathrm{pABW} 2 / \mathrm{repC} 1$ & $\begin{array}{l}\mathrm{Tc}^{\mathrm{r}} \text {, PCR-amplified } 1.3 \mathrm{~kb} \text { fragment of } \operatorname{rep} C \text { gene cloned } \\
\text { into Pst } \mathrm{I} \text { site of } \mathrm{pABW} 2 \text { under control of } \mathrm{P}_{\text {lac }}\end{array}$ & This work \\
\hline $\mathrm{pABW} 2 / \mathrm{repC} 2$ & $\begin{array}{l}\mathrm{Tc}^{\mathrm{r}}, \mathrm{pABW} 2 \text { with cloned } r e p \mathrm{C} \text { gene in opposite } \\
\text { orientation to that in } \mathrm{pABW} 2 / \mathrm{repC} 1\end{array}$ & This work \\
\hline pRK2013 & $\mathrm{Km}^{\mathrm{r}}$, helper plasmid carrying $\mathrm{RK} 2$ tra genes & Ditta et al. (1980) \\
\hline
\end{tabular}




\section{RESULTS AND DISCUSSION}

\section{Identification of the second replicator region of plasmid pTAV1}

Previously we constructed two mini-replicons carrying a $30 \mathrm{~kb}$ EcoRI (pTAV110) or $4 \mathrm{~kb}$ Sall-PstI (pTAV202) restriction fragment of the plasmid pTAV1 (Bartosik et al., 1995), and the appropriate form of the $\mathrm{Km}^{\mathrm{r}}$ cassette $(1 \cdot 3 \mathrm{~kb})$. The mini-replicon pTAV202 was not stably maintained in P. versutus cells, whereas pTAV110 was as stable as the parental pTAV1. After 30 generations of growth under non-selective conditions, $100 \%$ of cells tested carried pTAV110, while only $4 \%$ carried pTAV202. We cloned several restriction fragments of pTAV110 which were not included in pTAV202 and tested their influence in cis on this unstable replicon to identify the region responsible for stability. For this purpose we used the hybrid plasmid pTAV202/18 (Bartosik et al., 1995), which carried the $4 \mathrm{~kb}$ replicator region of pTAV202 cloned into the multiple cloning site of the $E$. coli-specific vector $\mathrm{pBGS18}$ and showed a similar degree of stability $(2 \%)$ to pTAV202. Insertion of the $16 \cdot 1 \mathrm{~kb}$ $B c l$ restriction fragment of pTAV110 into pTAV202/18 (pTAV202/18/16; Fig. 1 ) resulted in stabilization of the plasmid, indicating that this DNA fragment encoded mechanisms which enabled the stable maintenance of pTAV202. Plasmid pTAV202/18/16 was digested with $K p n I$ and religated to make a considerable deletion $(13.6 \mathrm{~kb})$ in the 'stabilizing' fragment. We found a class of $P$. versutus $\mathrm{Km}^{\mathrm{r}}$ transformants carrying a plasmid
(pTAV301; Fig. 1) with a different restriction pattern than expected. Detailed restriction analysis showed that the plasmid was composed of the excised region of the putative 'stabilizing' fragment (equipped with the $\mathrm{Km}^{\mathrm{r}}$ cassette). Therefore, pTAV1 carries a second separable replication system designated pTAV301 $(13.6 \mathrm{~kb})$. Hybridization analysis proved that both replicons (pTAV301 and pTAV202) are present on different BclI restriction fragments of the parental pTAV1 (as well as of pTAV110) and do not cross-hybridize (data not shown).

To identify the minimal region of pTAV301, the plasmid was digested with SalI and, after ligation, was electroporated into P. versutus UW225 (pTAV1-). We obtained $\mathrm{Km}^{\mathrm{r}}$ transformants carrying the mini-replicon pTAV310 containing the $9 \mathrm{~kb}$ Sall fragment of pTAV301. A partial digestion of pTAV310 with PstI, followed by ligation and further electroporation, enabled selection of transformants carrying a $5.6 \mathrm{~kb}$ plasmid, designated pTAV320 . This replicon was composed of two $(2 \cdot 2$ and $2 \cdot 1 \mathrm{~kb})$ PstI restriction fragments (aligned as in pTAV310 and pTAV1) and a $\mathrm{Km}^{\mathrm{r}}$ cassette. We did not succeed in obtaining a replicative form of the plasmid composed of a single PstI restriction fragment of pTAV 320 and $\mathrm{Km}^{\mathrm{r}}$ cassette. Further analysis suggested that pTAV320 might constitute a minimal replicon of a second pTAV1 replicator region. The construction of pTAV320 is shown in Fig. 1.

Detailed restriction analysis of pTAV203, pTAV320 and
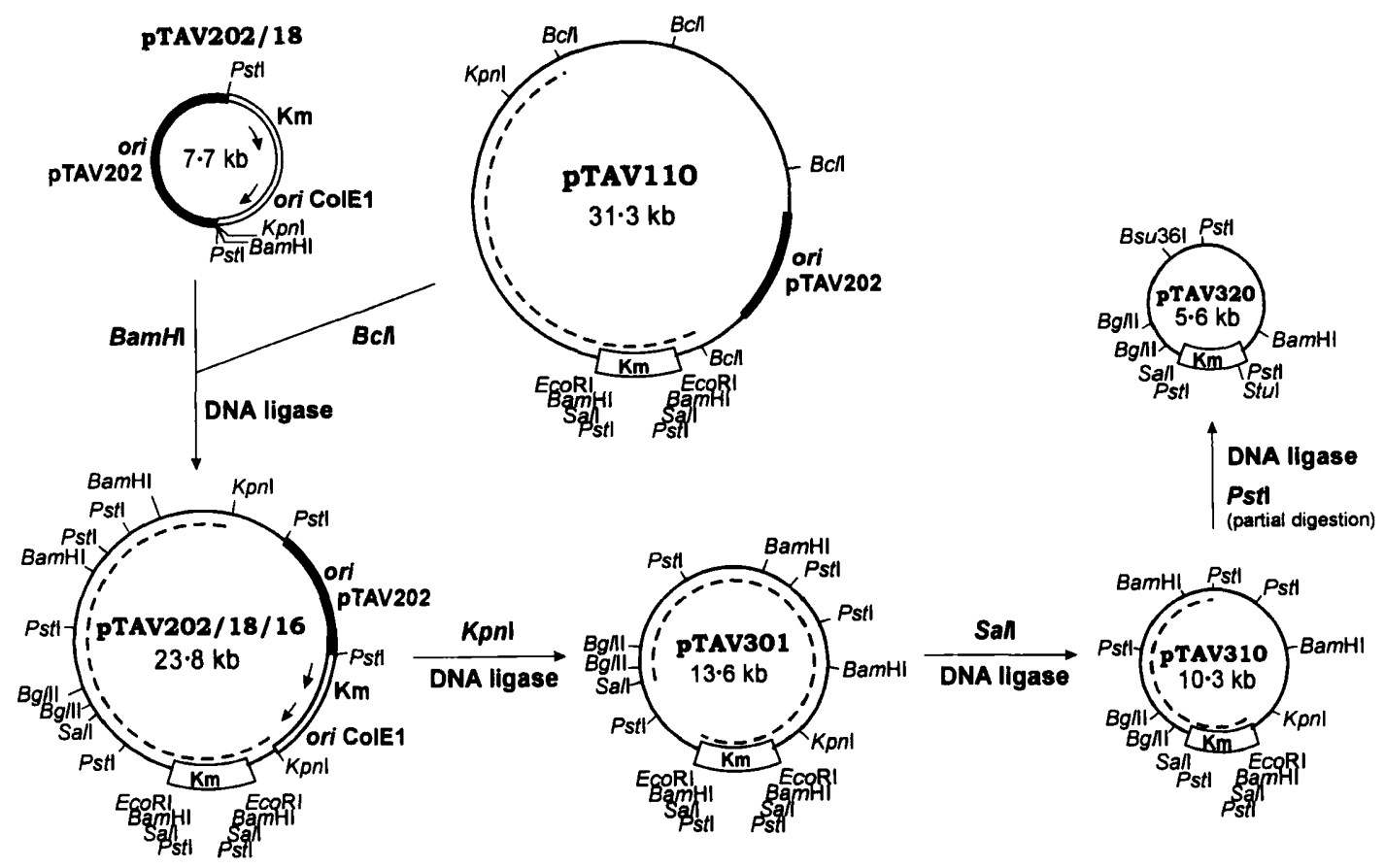

Fig. 1. Construction of the minimal replicon pTAV320. See Results and Discussion for explanations. The regions that contribute to the next stage of construction are marked with dashed lines. The $\mathrm{Km}^{\mathrm{r}}$ cassette is marked as a box. Only restriction sites important for construction are shown. The sites for Bsu36I and Stul are given only for pTAV320. Plasmids are not drawn to scale. 


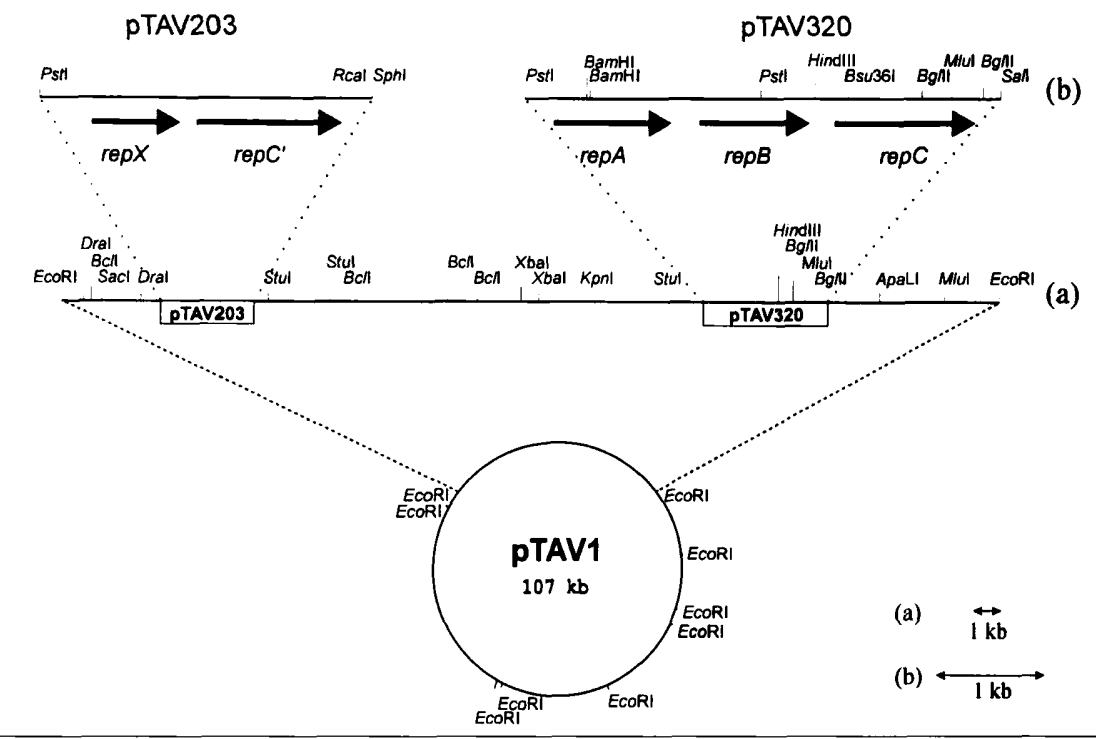

Fig. 2. Localization of the two identified replicator regions on PTAV1. The enlargements show (a) a detailed restriction map of the $30 \mathrm{~kb} E c o R I$ restriction fragment carrying the two identified replicator systems of PTAV1 and (b) restriction map and molecular organization of pTAV203 (Bartosik et al., 1997a) and pTAV320. The orientation of ORFs is indicated by arrows.

pTAV110 localized both replication systems on a single $30 \mathrm{~kb}$ EcoRI restriction fragment of pTAV1 (present in pTAV110) as shown in Fig. 2.

\section{Nucleotide sequence of pTAV320}

To examine the gene organization of the replicator region, the complete nucleotide sequence of the minimal replicon pTAV 320 was determined. Double-strand sequencing of pTAV320 covering the whole $4338 \mathrm{bp}$ sequence revealed the presence of three large, clustered ORFs, with identical transcriptional polarity, encoding proteins of approximately 35 (RepA), $36(\mathrm{RepB})$ and 47 (RepC) $\mathrm{kDa}$ (Fig. 2b). With the use of TFASTA (GCG software), we found that the putative translation products of pTAV320 show a significant and very similar level of homology to corresponding RepA, RepB and RepC proteins of pTiB6S3 of $A$. tumefaciens (Tabata $e t$ al., 1989), pRiA4b of A. rhizogenes (Nishiguchi et al., 1987), pRL8JI of Rhiz. leguminosarum (Turner \& Young, 1995), p42d of Rhiz. etli (Ramírez-Romero et al., 1997) and pNGR234a of Rhizobium sp. NGR234 (Freiberg et al., 1997). For example, the similarity/ identity between pTAV320 and the corresponding translation products of $\mathrm{pTiB} 6 \mathrm{S3}$ were: $\operatorname{RepA}, 71 / 56 \%$; RepB, 52/32\% ; and RepC, 61/37\%. RepA and RepB also have some similarity with several proteins encoded by non-rep $A B C$-type replicons (e.g. F and P1 plasmids; Williams \& Thomas, 1992) and involved in partitioning, which may suggest a role for RepA and RepB in pTAV320. In addition, RepB shows similarity with ParB, a chromosome-partitioning protein of Caulobacter crescentus (Mohl \& Gober, 1997). RepC of pTAV320 shows statistically significant degrees of similarity with the essential replication proteins RepC of pRmeRG4a of S. meliloti ( $29 \%$ identity) and RepC $C^{\prime}$ of pTAV203, the second pTAV1 replication system $(27 \%$ identity).

In addition to the sequence similarities, these genes are similar in size and structural organization in pTAV320 to those of other $r e p A B C$-type replicons. Thus pTAV320 appears to be the first example of this type of replicon found outside Agrobacterium and Rhizobium. Phylogenetic analysis of $\mathrm{RepC}$ proteins from replicons from the genera Agrobacterium, Rhizobium and Paracoccus indicated that RepC of pTAV320 is the most divergent of this group (data not shown).

In all published repABC-type replicons there is a short, highly conserved intergenic sequence (igs) situated between the repB and repC genes. In the case of $p R i A 4 b$ and $\mathrm{pTiB} 6 \mathrm{~S} 3$ this intergenic region was shown to express incompatibility towards the parental plasmid. It was suggested that the origin of replication (oriV) might be located within this region (Tabata et al., 1989). The multiple alignments of the igs sequences of different rep $A B C$-type plasmids are shown in Fig. 3. Although the igs of pTAV 320 is more divergent, all the conserved motifs are visible. The highest degree of identity was observed in two regions. One of them contains an A/Trich sequence followed by a $\mathrm{G} / \mathrm{C}$-rich region (AAAAGAAAAAGGCCCC), which in the case of pTAV320, pTiB6S3 and pNGR234a is identical. This motif is also present between ORF2 and RepC in pRmeGR4 of $S$. meliloti and in the intergenic region between RepX and RepC' of pTAV203, which are non-repABC-type replicons, but is less conserved in the latter one (ATAAGTAAACCCCCC). In all cases this motif was followed by a potential stem-loop structure. The second most conserved region of the igs of pTAV320 and other repABC-type replicons (TTTGCCT) was located upstream of the repC gene. The motifs present in the igs sequence might be involved in regulation of repC transcription or act as a target for as yet unidentified regulatory proteins which could be involved in incompatibility functions (Ramírez-Romero et al., 1997).

As has been observed in this type of replicon, there are two purine-rich regions, one preceding $r e p A$ and the other $r e p C$, analogous in sequence and position to the 


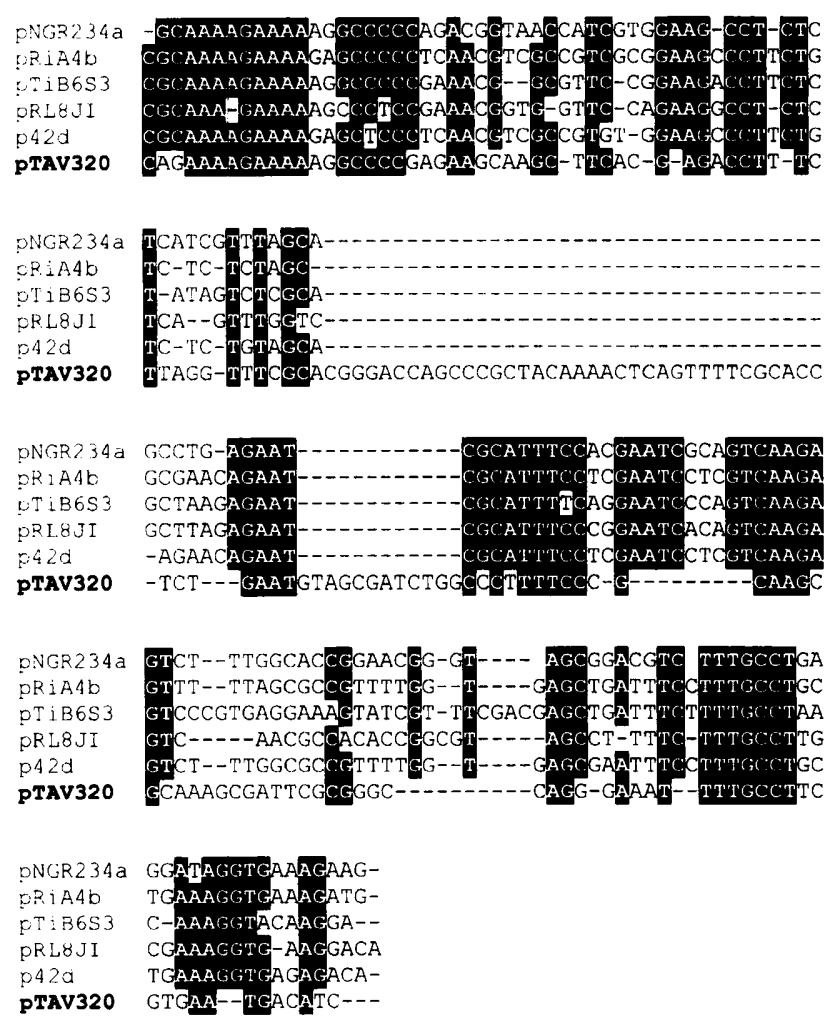

Fig. 3. Multiple alignments of the intergenic sequences (igs) of different repABC-type replicons: pNGR234a of Rhizobium sp. NGR234; pRiA4b of $A$. rhizogenes; pTiB6S3 of $A$. tumefaciens; pRL8JI of Rhiz. leguminosarum; p42d of Rhiz. etli; and pTAV 320 of $P$. versutus. Alignment of at least five identical residues is shown on a black background. Hyphens indicate gaps introduced to maximize the alignment.

ribosome-binding sites preceding translated reading frames of enteric bacteria (Shine \& Dalgarno, 1975). No sequence homologous to the -35 or the -10 promoter regions of E. coli was found upstream of the genes. The lack of a typical terminator structure within the pTAV320 sequence suggests that $r e p A, r e p B$ and $r e p C$ might constitute a common transcriptional unit. Further transcriptional analysis will help to elucidate the organization of the three loci.

\section{Host range}

The host range for pTAV1 has not yet been determined for lack of a convenient means of its transfer. pTAV1 is neither conjugative nor mobilizable. Its large size, low efficiency of electroporation and restriction barrier in many bacterial species make the use of transformation or electroporation ineffective. We have previously shown that the host range of pTAV202 is not limited to $P$. versutus and it can be maintained in $P$. denitrificans KL1 and A. tumefaciens LBA1010 (Bartosik et al., 1997a). We successfully electroporated pTAV320 into both the above strains. As with pTAV202, pTAV320 does not replicate in E. coli.
To overcome the restriction barrier, we constructed a convenient mobilizable hybrid plasmid, composed of an E. coli-specific mobilizable pABW2 vector and a $5.6 \mathrm{~kb}$ Stul restriction fragment carrying the replication system of pTAV320 together with a $\mathrm{Km}^{\mathrm{r}}$ cassette. The hybrid plasmid, designated pABW320, was introduced via triparental conjugal mating to Rhiz. etli CE3, Rhiz. leguminosarum 1062 and Rhod. sphaeroides 2.4.1R. This shows that pTAV320 is not a narrow-host-range replicon and can be maintained in several genera belonging to the $\alpha$ subclass of the Proteobacteria. pTAV320 (or pABW320) showed high levels of stability $(92-100 \%)$ in all host strains tested and similar to that in $P$. versutus UW225. The preliminary data for $\mathrm{PABW} 202$ suggest the same host range for both pTAV1 minireplicons.

\section{Functional characterization of pTAV320}

Plasmid replication. It was suggested that in the $\operatorname{rep} A B C$ type replicons, repC encodes the main replication protein. Mutations introduced into this gene completely abolished the ability to replicate (Tabata et al., 1989). The same mutational effect was observed in the case of pTAV320. It was not possible to obtain a replicon with a deletion of the $596 \mathrm{bp} \mathrm{BglII}$ fragment covering a terminal part of the repC gene (Fig. 2b), while mutations generated in repA or repB did not prevent replication. This proves that neither RepA nor $\operatorname{RepB}$ are indispensable for the initiation of replication. Deletion of a $32 \mathrm{bp} \mathrm{BamHI}$ fragment of repA of pTAV320 (pTAV321) or insertion of the $1.3 \mathrm{~kb} \mathrm{Km}^{\mathrm{r}}$ cassette into the PstI site of repB (pTAV322) affected stability of the mutated replicon. The stability of pTAV321 or pTAV322 was much reduced, and only 8 or $17 \%$ of the bacterial population, respectively, carried the plasmids (after 30 generations of growth under non-selective conditions), while pTAV320 was stably maintained $(98 \%)$ in $P$. versutus cells. A similar mutational effect was previously observed for repA or repB genes of pRiA4b or pTiB6S3 (Tabata et al., 1989).

We have previously shown that in pTAV202 the point of replication initiation (oriV) is located within the rep $\mathrm{C}^{\prime}$ gene. Because RepC' of pTAV202 and RepC of pTAV320 show homology and appear to play a similar role in replication, we assumed that oriV might be similarly located in pTAV320. To investigate this hypothesis, the $1.2 \mathrm{~kb}$ fragment carrying only the coding region for RepC with a putative ribosome-binding site was amplified by PCR and the fragment cloned, in two orientations (pABW2/repC1 and pABW2/repC2), into the PstI site of $\mathrm{pABW} 2$. The resulting transformants were used as donors in triparental mating. We obtained $T c^{r}$ transconjugants in $P$. versutus UW225 only with $\mathrm{pABW} 2 / \mathrm{repC} 1$, in which repC was placed under transcriptional control of the $\mathrm{pABW} 2$ lac promoter. This confirmed that $\mathrm{RepC}$ is sufficient for the initiation of replication and proved that oriV is located within its coding sequence. The sequence similarity between RepC and $\mathrm{RepC}^{\prime}$ of pTAV1 and localization of oriV within 


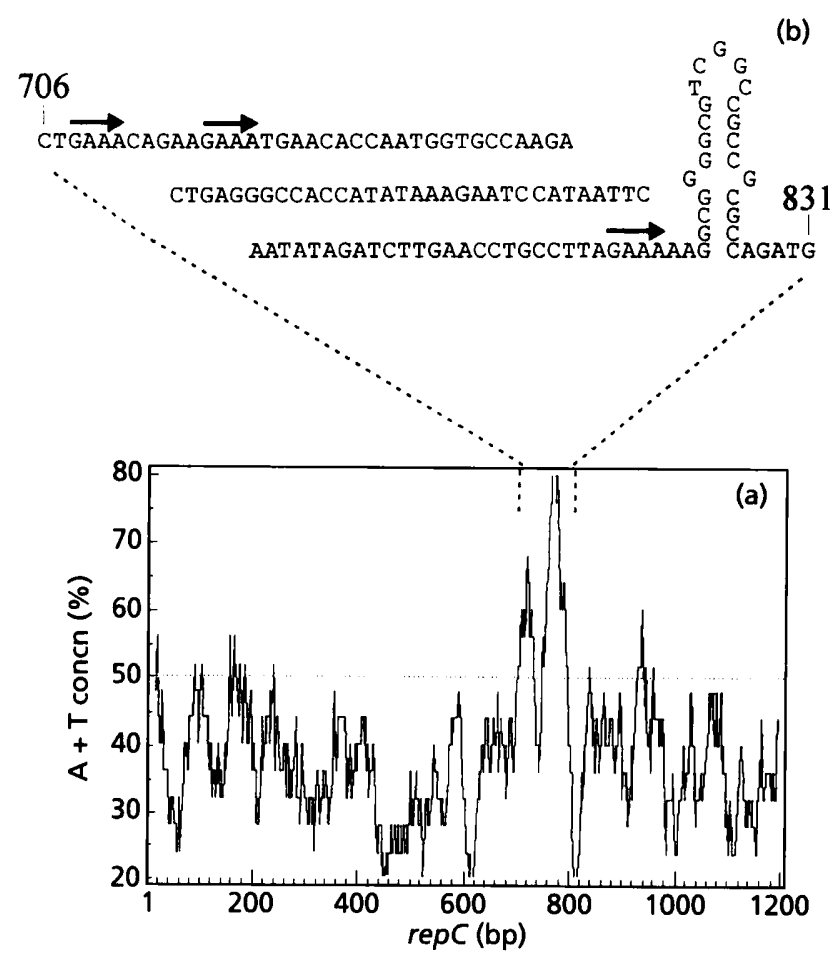

Fig. 4. Sequence and localization of the A/T-rich region of the repC gene of pTAV 320 . (a) $A+T$ profile of the repC gene (1211 bp). (b) Sequence of the region containing the putative origin of replication. Horizontal arrows indicate 4 bp repeats.

both genes suggest a close relationship between these replication systems.

Within the central region of the $\operatorname{rep} C$ sequence we found a $97 \mathrm{bp} \mathrm{A} / \mathrm{T}$-rich region of $63 \% \mathrm{~A}+\mathrm{T}$ (compared to $39 \%$ overall pTAV320 DNA), containing three short (4 bp) repeats, followed by a potential stem-loop structure (Fig. 4). We speculate that the region extending from position 706 to 831 of the repC gene might contain the origin of replication. A similar region and structure, localized in an analogous position, has been previously suggested to form the oriV of megaplasmid pMOL28 of Ralstonia eutropha (Alcaligenes eutrophus) and it was suggested that in this case a transcriptional activation of the origin may occur (Taghavi et al., 1996).

Partition functions. In the case of well-characterized partitioning systems it was possible to construct a specific cassette, encoding two proteins and a cis-acting centromere-like sequence, which could provide stability functions when cloned into other heterologous replicons (Williams \& Thomas, 1992). To analyse the tentative partitioning nature of RepA and RepB we tested their influence in cis on pTAV202, the other unstable replicon of pTAV1. pTAV202 was lost during incubation under non-selective conditions and after 30 generations of growth only approximately $4 \%$ of the bacterial population harboured the plasmid. For the analysis we chose the mobilizable hybrid plasmid pABW202 (Table 1; Fig. 5 ), which enabled cloning in $E$. coli and efficient conjugational transfer of the constructs into $P$. versutus. As a control the complete, non-mutated genome of pTAV320 linearized by StuI digestion was cloned into the blunted HindIII site of pABW202 (downstream of the $4 \mathrm{~kb}$ insert of pTAV202 to minimize a possible influence of lac promoter on expression of the cloned rep genes). As expected the derived pABW2022 (carrying the two functional replicator regions of pTAV1) showed a similar level of stability to pTAV320 (Fig. 5). Assuming that the centromere-like sequence might be located along the highly conserved igs sequence we cloned the $B s u 36 \mathrm{I}-B g l \mathrm{II}(4.3 \mathrm{~kb})$ restriction fragment of pTAV320 carrying repA, repB, the complete igs region, $70 \mathrm{bp}$ of repC and the $\mathrm{Km}^{\mathrm{r}}$ cassette (enabling selection of recombinant clones). The resulting plasmid, designated pABW2021, was not more stable than pABW202 itself (Fig. 5) and its stability was even decreased. This suggests that the cloned fragment did not contain a complete partitioning system (probably lacking the centromere-like sequence).

To test the possibility that the sequence required for partitioning might be localized along $\operatorname{rep} C$, we cloned into pABW202 the following fragments: (1) $5 \mathrm{~kb} \mathrm{BglII}$ fragment from $\mathrm{pTAV} 320$ carrying $\operatorname{repA}$, $\operatorname{rep} B$, igs sequence and part of the repC gene $(779 \mathrm{bp}) ;(2)$ the entire pTAV320 $(5.6 \mathrm{~kb}$ ) linearized with Bsu36I (which inactivates its replication activity). The resulting plasmids were designated pABW2023 and pABW2024, respectively. pABW2023 showed a similar level of stability to pABW2021, whereas pABW2024 was more stable $(51 \%)$ than pABW202 itself $(1.5 \%)$. Thus only the presence of the whole sequence of pTAV320 (plasmid pABW2024) was able to increase the stability of pTAV202. Construction and stability of all recombinant clones are shown in Fig. 5. In a further control experiment all the above-mentioned restriction fragments of pTAV320 were also cloned into pABW2, but only one, as expected, carrying the linearized genome of pTAV320 as a $5.6 \mathrm{~kb}$ StuI fragment (pABW320) was shown to be able to replicate in P. versutus (data not shown).

These results suggest that partition functions require the presence of an intact repC gene. To confirm the role of $r e p C$ in partition we chose the fully stable pTAV110, a composite mini-replicon which carries the two replicator regions on a single EcoRI $(30 \mathrm{~kb})$ restriction fragment of pTAV1 (Fig. 2a). Introduction of the $596 \mathrm{bp}$ BglII deletion into pTAV110 did not abolish the replication function of the resulting plasmid, pTAV112 (indicating that the second replicator region from pTAV202 carried out replication), but reduced its stability $(<1 \%)$ to that of pABW 2023 .

These results confirm that stable maintenance of the pABW202 recombinant plasmids, pTAV110 and probably pTAV1 is dependent on the presence of pTAV320, which carries the complete partitioning system. Our observations show that the coding sequence for the $r e p C$ gene is important for plasmid maintenance and that cisacting sequences for the origin of replication and the centromere-like sequence might be located within it. 


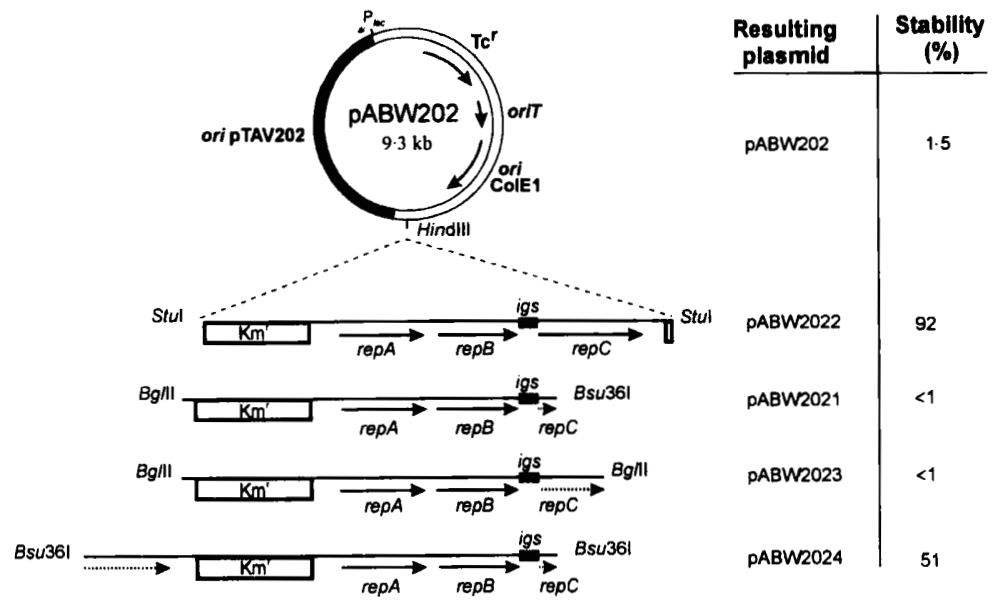

$\mathrm{I} \mathrm{kb}$
Fig. 5. Analysis of the stabilizing activity of different fragments of the pTAV 320 replicon on pTAV202 present in the recombinant plasmid PABW202. See text for explanations. The truncated repC gene is marked as a dotted arrow. Stability was determined as described in Methods.

\section{Incompatibility of pTAV1 mini-replicons}

Incompatibility of the two mini-replicons pTAV202 and pTAV320 towards the native pTAV1 was tested via electroporation of each mini-replicon into $P$. versutus UW1 carrying native pTAV1. The frequency of electroporation into this strain was $10^{3}$ times lower than into UW225 (pTAV1-less strain) and none of the $\mathrm{Km}^{\mathrm{r}}$ transformants carried the $\mathrm{pTAV} 1$ plasmid, which shows that both mini-replicons expressed strong incompatibility towards the residing parental plasmid. We also tested the possibility of the coexistence of the two minireplicons in trans in $P$. versutus cells. For this purpose pTAV320 was electroporated into $P$. versutus UW225 carrying pTAV202T $\left(a \mathrm{Tc}^{\mathrm{r}}\right.$ derivative of pTAV202). The $\mathrm{Km}^{\mathrm{r}} \mathrm{Tc}^{\mathrm{r}}$ transformants carried both plasmids, which were able to coexist during growth under nonselective conditions and were lost from the population at the rates characteristic for the individual plasmids (data not shown). These results indicate that both replicons are compatible in trans and suggest that incompatibility towards pTAV1 might be caused by the existence of common regulatory elements for the two replicator regions which are challenged by the incoming mini-replicons.

Some studies on the compatibility of pTAV1 minireplicons with other plasmids present in $P$. denitrificans and $A$. tumefaciens have also been done. pTAV 320 expresses incompatibility towards pTiB6S3 of A.tumefaciens, indicating a very close relationship between these plasmids which naturally occur in different bacterial genera. Interestingly, pTAV1 mini-replicons showed a different incompatibility behaviour towards the $P$. denitrificans pKL1 plasmid $(\sim 100 \mathrm{~kb})$. pTAV320 expressed incompatibility towards pKL1 while pTAV202 could be maintained together with this plasmid (Jordan et al., 1997). However, we found the minireplicon pTAV110 to be fully compatible with pKL1, which suggests that in the presence of pKL1 the replication of the composite plasmid was carried by pTAV202. In this case the plasmid was fully stable, thus confirming the role of the partitioning system of pTAV320 in the stable maintenance of pTAV110 (and probably entire pTAV1).

\section{Concluding remarks}

pTAV1 is a composite plasmid and carries, on a single EcoRI restriction fragment, two replicator regions able to form separate mini-plasmids. The two replicons express incompatibility towards the parental plasmid but are compatible when present in trans in P. versutus UW 225 cells.

Sequence analysis of pTAV320 showed the presence of three ORFs similar in sequence and organization to $r e p A$, rep $B$ and repC found in rep $A B C$-type replicons of several Agrobacterium and Rhizobium plasmids. RepC was confirmed to be the major replicator protein and ori $\mathrm{V}$ was localized to the rep $\mathrm{C}$ gene. RepA and $\mathrm{RepB}$ are necessary for stable maintenance of the plasmid. However, only the presence of the whole sequence of pTAV320 (in its non-replicative form) could stabilize pTAV202 in cis. This suggests a role for $r e p C$ in plasmid partitioning.

pTAV320 can be maintained in several Paracoccus, Agrobacterium, Rhizobium and Rhodobacter strains but not in E. coli. Incompatibility of pTAV320 and pKL1 of $P$. denitrificans suggests that this type of replicon $(r e p A B C)$ might be widespread not only, as previously suggested, among species of Agrobacterium and Rhizobium, but also in other genera belonging to the $\alpha$ subclass of the Proteobacteria.

\section{ACKNOWLEDGEMENTS}

We thank Edyta Wysocka for assistance in determination of plasmid stability. We acknowledge P. J. J. Hooykaas, S. Kaplan, M. A. Ramírez-Romero and J. P. W. Young for providing the strains Agrobacterium tumefaciens LBA1010, Rhodobacter sphaeroides 2.4.1, Rhizobium etli CE3 and Rhizobium leguminosarum 1062, respectively. 


\section{REFERENCES}

Bartosik, D., Baj, J., Plasota, M., Piechucka, E. \& Wlodarczyk, M. (1993). Analysis of Thiobacillus versutus pTAV1 plasmid functions. Acta Microbiol Pol 39, 5-11.

Bartosik, D., Baj, J. \& Wlodarczyk, M. (1995). Construction and preliminary characterisation of mini-derivatives of large $(107-\mathrm{kb})$ cryptic plasmid of the sulphur bacterium Thiobacillus versutus. FEMS Microbiol Lett 129, 169-174.

Bartosik, D., Wlodarczyk, M. \& Thomas, C. M. (1997a). Complete nucleotide sequence of the replicator region of Paracoccus (Thiobacillus) versutus pTAV1 plasmid and its correlation to several plasmids of Agrobacterium and Rhizobium species. Plasmid 38, 53-59.

Bartosik, D., Bialkowska, A., Baj, J. \& Wlodarczyk, M. (1997b). Construction of mobilizable cloning vectors derived from pBGS18 and their application for analysis of replicator region of a pTAV202 mini-derivative of Paracoccus versutus pTAV1 plasmid. Acta Microbiol Pol 46, 379-383.

Bednarska, M., Jagusztyn-Krynicka, E. K., Popowski, J. \& Wlodarczyk, M. (1983). Extrachromosomal DNA in Thiobacillus versutus. FEMS Microbiol Lett 16, 183-185.

Bergquist, P. L., Saadi, S. \& Maas, W. K. (1982). Molecular homology and incompatibility in the IncF plasmid group. $J$ Gen Microbiol 128, 223-238.

Beringer, J. E. (1974). $\mathrm{R}$ factor transfer in Rbizobium leguminosarum. J Gen Microbiol 84, 188-198.

Birnboim, H. C. \& Doly, J. (1979). A rapid alkaline extraction procedure for screening recombinant plasmid DNA. Nucleic Acids Res 7, 1513-1519.

Ditta, G., Stanfield, S., Corbin, D. \& Helinski, D. R. (1980). Broad host-range DNA cloning system for Gram-negative bacteria: construction of a bank of Rhizobium meliloti. Proc Natl Acad Sci USA 77, 7347-7351.

Felsenstein, J. (1993). PHYLIP (Phylogeny Inference Package) version 3.5.1. Seattle: Department of Genetics, University of Washington.

Freiberg, C., Fellay, R., Bairoch, A., Broughton, W. J., Rosenthal, A. \& Perret, X. (1997). Molecular basis of symbiosis between Rbizobium and legumes. Nature 378, 394-401.

Hanahan, D. (1983). Studies on transformation of Escherichia coli with plasmids. $J$ Mol Biol 166, 557-580.

Helinski, D. R., Toukdarian, A. E. \& Novick, R. P. (1996). Replication control and other stable maintenance mechanisms of plasmids. In Escherichia coli and Salmonella: Cellular and Molecular Biology, pp. 2295-2324. Edited by F. C. Neidhardt and others. Washington, DC: American Society for Microbiology.

Hirsch, P. R., van Montagu, M., Johnston, A. W. B., Brewin, N. J. \& Schell, J. (1980). Physical identification of bacteriocinogenic, nodulation and other plasmids in strains of Rhizobium leguminosarum. J Gen Microbiol 120, 403-412.

Jordan, S. L., McDonald, I. R., Kraczkiewicz-Dowjat, A. J., Kelly, D. P., Rainey, F. A., Murrell, J. C. \& Wood, A. P. (1997). Autotrophic growth on carbon disulfide is a property of novel strains of Paracoccus denitrificans. Arch Microbiol 168, 225-236.

Koekman, B. P., Hooykaas, P. J. J. \& Schilperoort, R. A. (1982). A functional map of the replicator region of the octopine $\mathrm{Ti}$ plasmid. Plasmid 7, 119-132.

Kushner, S. R. (1978). An improved method for transformation of E. coli with ColE1-derived plasmids. In Genetic Engineering, pp.
17-23. Edited by H. B. Boyer \& S. Nicosia. Amsterdam: Elsevier/North-Holland.

Mercado-Blanco, J. \& Olivares, J. (1994). The large nonsymbiotic plasmid pRmeGR4a of Rhizobium meliloti GR4 encodes a protein involved in replication that has homology with the RepC protein of Agrobacterium plasmids. Plasmid 32, 75-79.

Mohl, D. M. \& Gober, J. W. (1997). Cell cycle-dependent polar localization of chromosome partitioning proteins in Caulobacter crescentus. Cell 88, 675-684.

Nishiguchi, R., Takanami, M. \& Oka, A. (1987). Characterization and sequence determination of the replicator region in the hairyroot-inducing plasmid pRiA4b. Mol Gen Genet 206, 1-8.

Noel, K. D., Sánchez, A., Fernández, L., Leemans, J. \& Cevallos, M. A. (1984). Rhizobium phaseoli symbiotic mutants with transposon Tn5 insertions. J Bacteriol 158, 148-155.

Perkins, J. B. \& Youngman, P. (1983). Streptococcus plasmid pAM $\alpha 1$ is a composite of two separable replicons, one of which is closely related to Bacillus plasmid pBC16. J Bacteriol 155, 607-615.

Ramirez-Romero, M. A., Bustos, P., Girard, L., Rodriguez, O., Cevallos, M. A. \& Dávila, G. (1997). Sequence, localization and characteristics of the replicator region of the symbiotic plasmid of Rhizobium etli. Microbiology 143, 2825-2831.

Sambrook, J., Fritsch, E. F. \& Maniatis, T. (1989). Molecular Cloning: a Laboratory Manual, 2nd edn. Cold Spring Harbor, NY: Cold Spring Harbor Laboratory.

Shine, J. \& Dalgarno, L. (1975). Determination of cistron specificity in bacterial ribosomes. Nature 254, 34-38.

Spratt, B. G., Hedge, P. J., te Heesen, S., Edelman, A. \& BroomeSmith, J. K. (1986). Kanamycin-resistant vectors that are analogues of plasmids pUC8, pUC9, pEMBL8 and pEMBL9. Gene 41, 337-342.

Suwanto, A. \& Kaplan, S. (1992). A self-transmissible, narrowhost-range endogenous plasmid of Rhodobacter sphaeroides 2.4.1: physical structure, incompatibility determinants, origin of replication, and transfer functions. J Bacteriol 174, 1124-1134.

Tabata, S., Hooykaas, P. J. J. \& Oka, A. (1989). Sequence determination and characterization of the replicator region in the tumour-inducing plasmid pTiB6S3. J Bacteriol 171, 1665-1672.

Taghavi, S., Provoost, A., Mergeay, M. \& van der Lelie, D. (1996). Identification of a partition and replication region in the Alcaligenes eutrophus megaplasmid pMOL28. Mol Gen Genet 250, 169-179.

Taylor, B. F. \& Hoare, D. S. (1969). New facultative Thiobacillus and re-evaluation of the heterotrophic potential of Thiobacillus novellus. J Bacteriol 100, 487-497.

Turner, S. \& Young, J. P. W. (1995). The replicator region of the Rhizobium leguminosarum cryptic plasmid pRL8JI. FEMS Microbiol Lett 133, 53-58.

Vieira, J. \& Messing, J. (1982). The pUC plasmids, an M13mp7derived system for insertion mutagenesis and sequencing with synthetic universal primers. Gene 19, 259-268.

Williams, R. \& Thomas, C. M. (1992). Active partitioning of bacterial plasmids. J Gen Microbiol 138, 1-16.

Wlodarczyk, M., Jagusztyn-Krynicka, E. K., Bartosik, D. \& Kalinowska, I. (1994). Electroporation of Thiobacillus versutus with plasmid DNA. Acta Microbiol Pol 43, 223-227.

Received 20 April 1998; revised 29 June 1998; accepted 10 July 1998. 